\title{
Photoacoustic in remote sensing
}

\section{Tomasz Borowski}

Tomasz Borowski, "Photoacoustic in remote sensing," Proc. SPIE 11442, Radioelectronic Systems Conference 2019, 1144211 (11 February 2020); doi: $10.1117 / 12.2565308$

SPIE. Event: Radioelectronic Systems Conference 2019, 2019, Jachranka, Poland 


\title{
Photoacoustic in remote sensing
}

\author{
Tomasz Borowski \\ tomasz.borowski@wat.edu.pl \\ Military University of Technology, gen. Sylwester Kaliski Str., 200-908 Warszawa, Poland
}

\begin{abstract}
One of the first applications of the photoacoustic effect included the wireless voice communication over long distances. Nowadays, the photoacoustic methods have laid the foundation for the operation of measuring instruments, used e.g. for contactless specification of the composition and spatial distribution of substances. Photoacoustic methods are also used in the ultra-sensitive spectroscopic examination of trace gases, e.g. for the detection of toxic substances, explosives, biological materials. Remote sensing applications, such as contactless imaging of material structures as well as biological in vivo structures are a manifestation of the extraordinary versatility of photoacoustic methods. Photoacoustic imaging is of very high resolution and contrast. Selected aspects of photoacoustics in remote sensing, especially the possibility of remote sensing of underwater objects have been presented with the history of photoacoustics development in the background.
\end{abstract}

Keywords: photoacoustic, optoacoustic, remote sensing, optical sonar

\section{INTRODUCTION}

The photoacoustic effect was discovered in the 19th century by Bell and Tainter, who, motivated by a desire to build a device for voice communication similar to a modern telephone, conducted numerous experiments and research. Their joint work resulted in many inventions and discoveries, mainly in the development of acoustic-optical and opto-acoustic transducers and a device for long-distance wireless sound transmission called the photophone [1-5].

In the 19th century, photophone was the first device through which it was possible to perform a long-distance two-way connection using free space optical communication. While testing various materials and configurations to find the most effective way of producing optical signals modulated by voice, as well as their transmission and subsequent sensing, the effect - later called the photoacoustic effect - was unintentionally discovered. Bell presented the information and comments on the observation of an intriguing effect of the acoustic interaction of light and matter to Röntgen and Tyndall, who almost immediately started further research of their own [6-7]. Röntgen's research into the sound produced by light-absorbing gases can be seen as the beginning of photoacoustic applications in remote sensing.

\subsection{Selected examples of applications of the photoacoustic techniques}

From the time of Bell's pioneering experiments and the works of Röntgen and Tyndall until the outbreak of World War II, only a few photoacoustics papers were published, the most important of which seems to be 1938 work of Viegerov [8]. It explores the possibility of applying the photoacoustic effect in the analysis of the air composition in terms of the sensing weakly absorbing substances in the optical range (i.e. highly diluted gas solutions). In turn, in his 1943 work, Luft demonstrated exceptionally high effectiveness of the photoacoustic technique in fast and safe sensing of very low concentrations of extremely poisonous combat gases, e.g. sarin, identified through its ppm concentrations[9]. Both works permanently included photoacoustics in the area of photoacoustical spectroscopic techniques, abbreviated as PAS (Photo Acoustic Spectroscopy).

The collected gas samples were introduced into the interior of the photoacoustic device, into the so-called photoacoustic cell, inside of which they were exposed to a beam of light.

The beam of light had the frequencies selected for the strongest absorption by the substance searched for in the gas sample. The photoacoustic signal obtained in this way depended on the type and concentration of the component detected in the mixture. [1

Radioelectronic Systems Conference 2019, edited by Piotr Kaniewski, Jan Matuszewski, Proc. of SPIE Vol. 11442, $1144211 \cdot$ C 2020 SPIE · CCC code: 0277-786X/20/\$21 · doi: 10.1117/12.2565308 


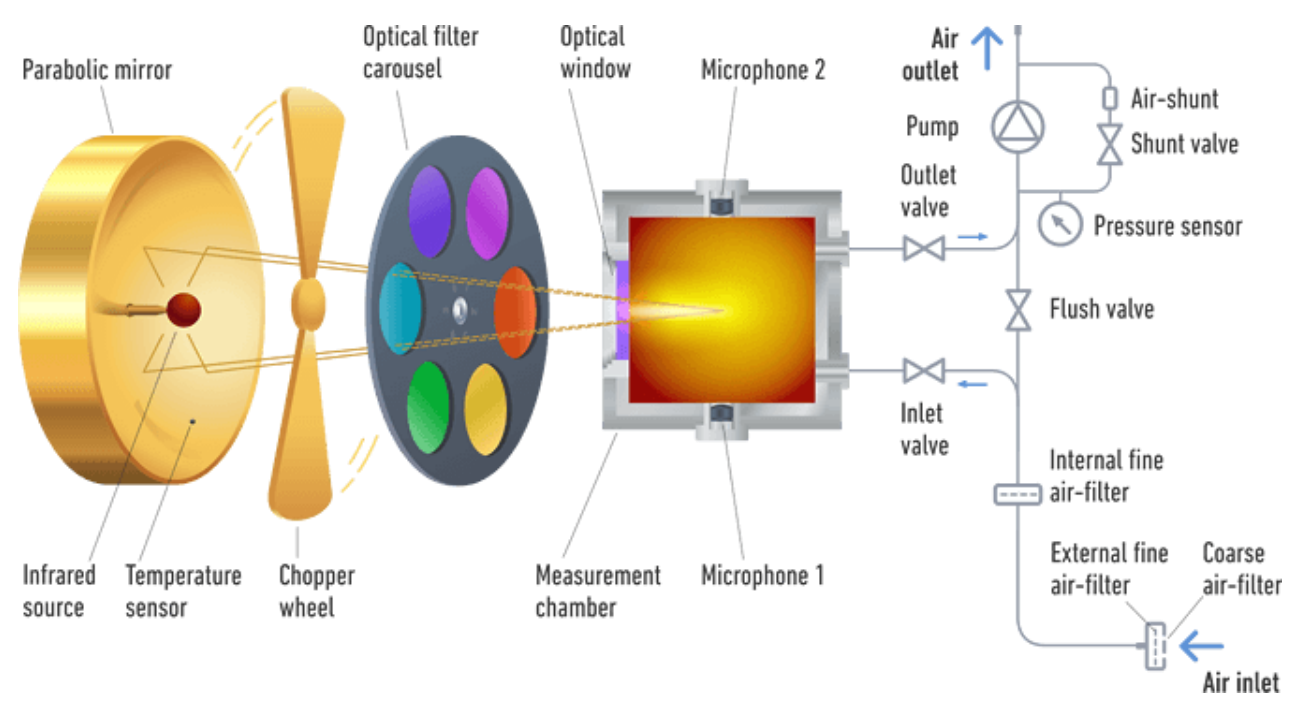

Figure 1. Measurement principle of Photoacoustic Gas Monitoring [40]

The intensity of the light beam was modulated with an optical chopper and the effects of pressure change induced in the photoacoustic chamber via a photoacoustic effect were received with a microphone. Nowadays, other photoacoustic signal transducers are also used, e.g. piezoelectric micro-tuning forks (QEPAS-type systems) [10-12], optical interference transducers and fiber optic transducers [13]. The aforementioned instruments are widely used in laboratory measurements.

Initially, all photoacoustic devices analysing trace gases required collecting, transporting and placing the analysed samples inside the device (the photoacoustic cell). Due to this factor such instruments could not be used to detect the substance sought directly in the free space and therefore did not fully meet the definition of a remote-sensing device.

The possibility of ultra-precise and fully photoacoustic remote sensing (directly in the free space over a distance) was demonstrated in the works of Brassington (1982) [15], Yönak and Dowling (2002) [16], Huang and Dowling (2003) [17] and $\mathrm{Ng}$ (2004) [18]. Possible solutions have proven that it is possible to detect micro chemical leaks directly into the atmosphere (even below $\mu 1 /$ month), which can escape e.g. through micro cracks and micro leaks in tanks or pipelines.

In order to detect leaks of the compound of interest, the monitored space was treated by laser pulses with the wavelength precisely adjusted to the absorption spectrum of the compound of interest. If molecules of the compound were found in the path of such a laser beam, the photoacoustic signal induced by the laser pulse was received by directional microphones or by multiple microphone systems arranged in a line or matrix of microphones. Further development in photoacoustic remote sensing progressed with the development of optical beam forming methods, techniques of modulation and scanning of space with optical beams, as well as the development of highly-sensitive acoustic-electric transducers, low-noise electronic amplifiers and signal processing methods.

Nowadays, the photoacoustic effect has laid the foundation for ultra-sensitive, spectroscopically selective and nondestructive methods of contactless specification of the composition and spatial distribution of compounds.

Photoacoustic techniques are suitable for extremely low concentrations of the component in a mixture, even at the level of $10^{-12}$. This feature in remote sensing is particularly desirable in the selective detection of trace concentrations of e.g. drugs, toxic substances, isotopes, explosives, blood oxygen saturation, to non-invasive glucose measurement, biological materials, e.g. anthrax spores, which may be a part of e.g. improvised terrorist loads [19-23].

Photoacoustic tomography uses a pulsed laser beam technique that provides light pulses to generate sound waves in a sample, which is then detected using an ultrasonic detector. Images of structures through which the generated sound waves pass are determined on the basis of calculations, e.g. [24]

Photoacoustic tomography uses a pulsed laser beam that provides light pulses to generate sound waves in the sample, which are then detected using an ultrasonic detector. Images of structures through which the generated sound waves pass are determined by calculations. 
Currently, this technique allows dynamic imaging of tested sample structures with the frequency of recording tomographic cross-sections up to several thousand frames per second.

The exceptional versatility of photoacoustic methods is evidenced by the possibility of obtaining high quality in vivo tissue images. The images obtained are characterized by very high resolution, contrast and depth of field. They are obtained from the acoustic response of light treated structures absorbing ultra-short optical pulses.

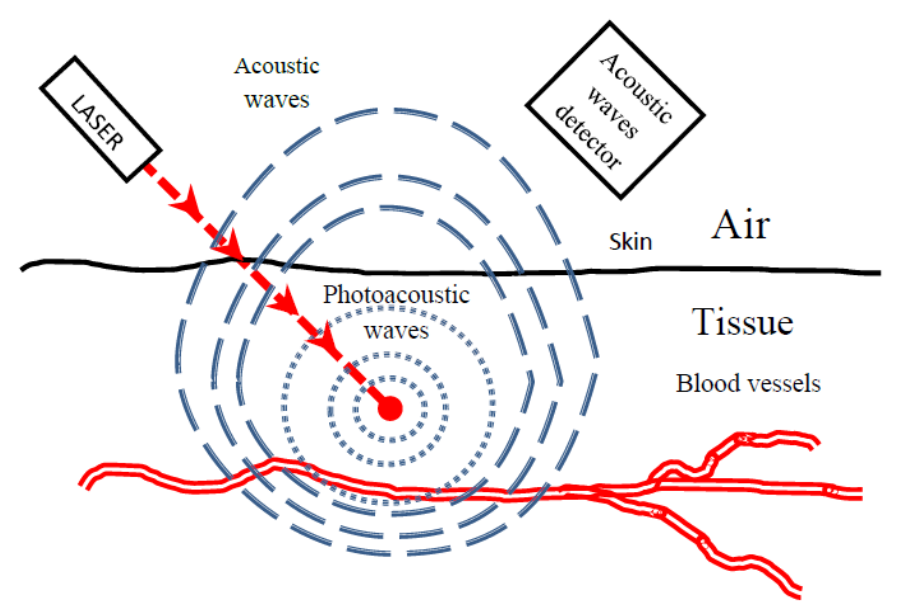

Figure 2. An example of how photoacoustic tomography works.

he high quality of images obtained with a contrast photoacoustic scanner is attractive because it allows selective observation of cells and tissue structures, allowing, for example, to study their metabolism, growth, motions and their other behaviours, even in vivo [23-24].

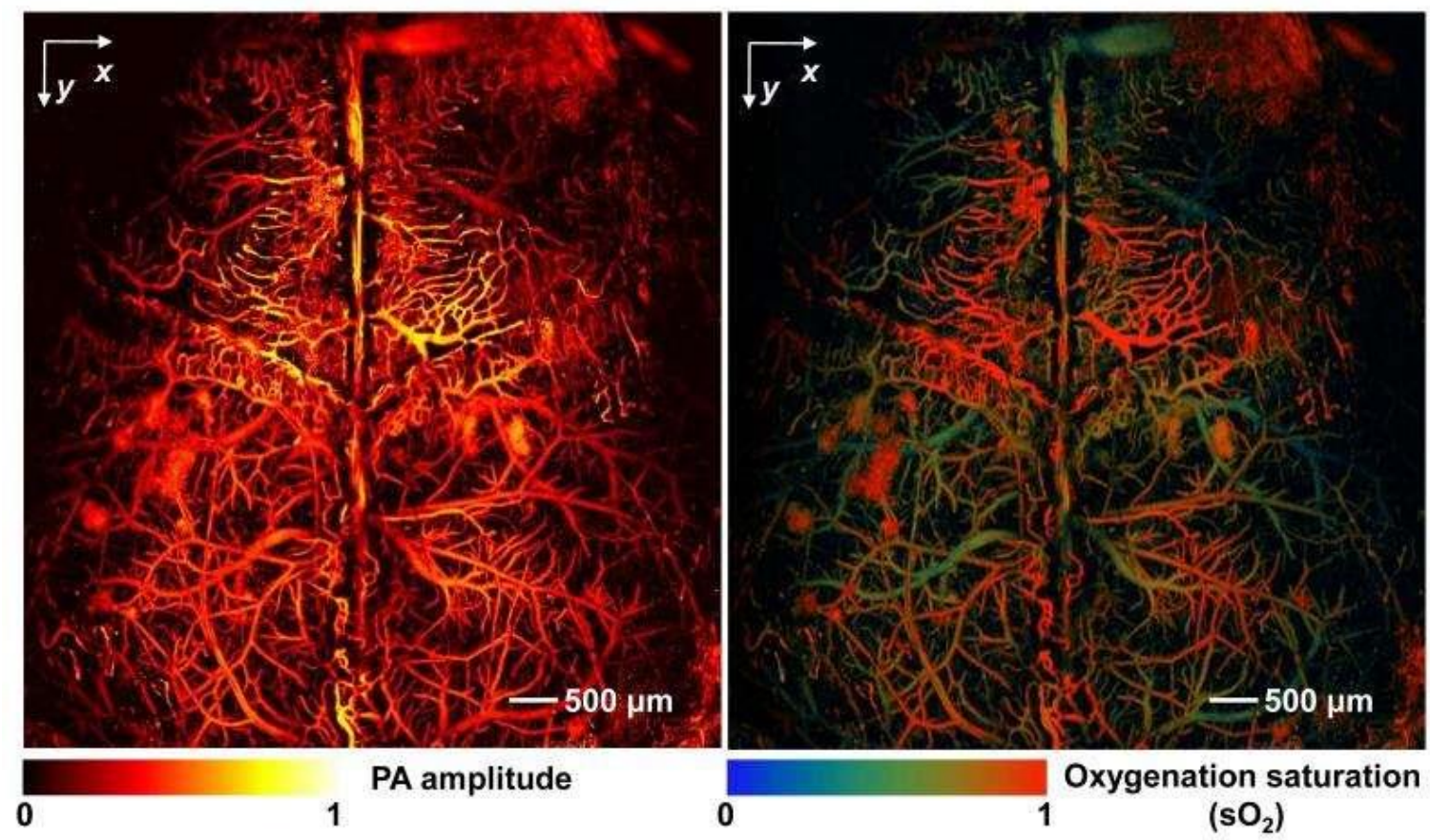

Figure 3. An example of imaging mouse brain tissues in vivo by photoacoustic microscopy technique [24]. 
An example of the quality of tissue imaging by photoacoustic microscopy reveals tissue oxygenation and their details structure with micrometre resolution [38].

The aforementioned examples of applications of the photoacoustic effect show that the idea of including photoacoustic methods in the remote sensing of underwater objects does not seem to be too exotic, but it even appears as a consequence of the development of remote sensing techniques.

\subsection{Selected problems with remote-sensing of underwater objects}

The issue of detecting underwater objects, particularly fully-submerged submarines, appears to be one of the greatest challenges of modern remote sensing.

The career of radar techniques that have developed spectacularly in relation to air remote-sensing has suggested the possibility of achieving similar success in the detection of underwater objects by radio waves.

Research carried out in this area quickly showed that due to the properties of water suppressing radio waves (inversely proportional to the electromagnetic wavelength), using them for underwater remote sensing using is problematic.

The VLF range (10-30kHz) is limited to relatively thin, near-surface water layers - typically up to 10-15 meters and short distances. On the other hand, the usability of the extremely low frequency ELF $(3-30 \mathrm{~Hz})$ range of waves, which penetrate depths greater than one hundred meters, also remains theoretical.

Radio waves with extremely low frequencies (i.e. extremely long wavelengths) are not adequate for detecting objects with dimensions much smaller than their wavelengths. For example, the length of the world's largest known submarine, the "Typhoon" (approx. $175 \mathrm{~m}$ ) is negligible compared to the EFL wavelengths (well over 10,000 km).

Systems operating in the VLF and ELF ranges are also limited by the huge dimensions of antennas $(50-70 \mathrm{~km})$, which due to their size are easy and vulnerable to damage.

Until recently, submarines were mainly detected by listening, sonar buoys, hydrophone systems and towed arrays sensor systems. Each of these techniques, however, does not meet the expectations of effective detection of the object and underwater remote sensing (i.e. spotting of the object).

Another technique used to detect earthquakes, submarines and monitor their movement, e.g. via strategic sea lanes and straits, are fiber optic sensors organized in sensor networks or sensor fields covering the seabed. Surveillance of large areas - even seas and parts of the oceans - became possible through the existing undersea fibrer optic telecommunication network. However, the aforementioned systems are available only to some countries or owners and operators of undersea fibre-optic infrastructure [24-29].

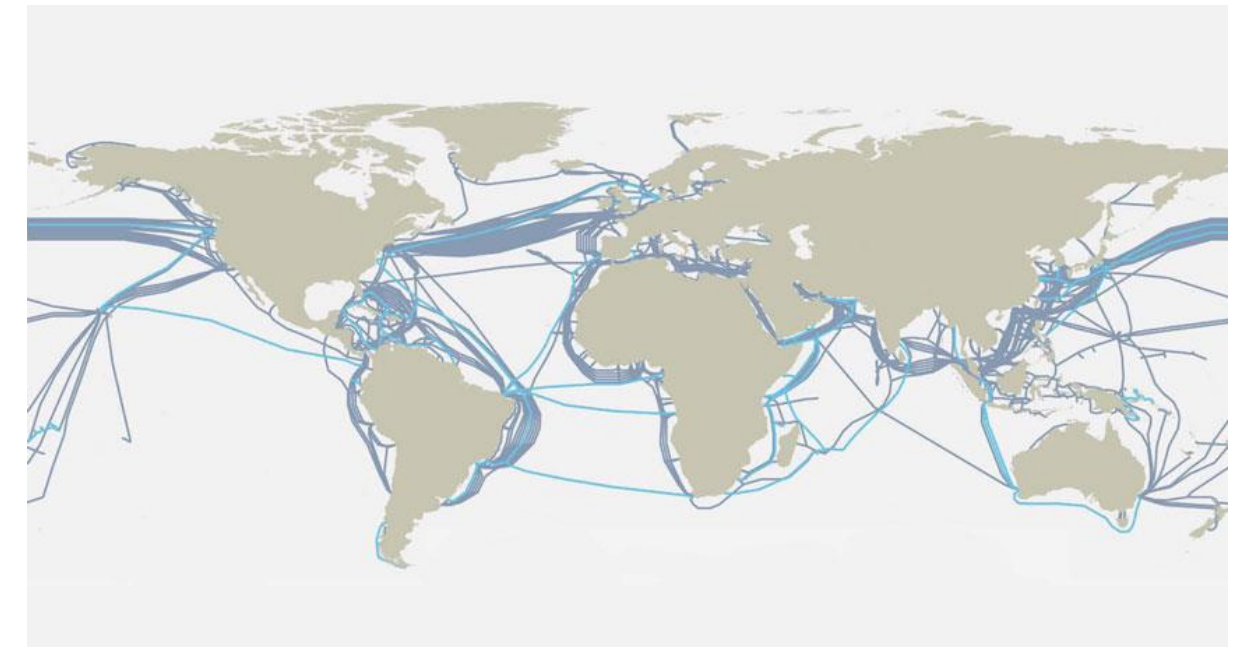

Figure.4. The foundation of submarine sensing technique based on the optical response of the sea surface [41] 
The global network of submarine fiber-optic cables may serve as a sensor for monitors motion of the ocean depth water and floor. The fiber optic net around the world could deliver data on detection undersea earthquake as well as a submarine detectors. Existing cables are shown in purple; planned cables are in blue.

Patent No. US3501639 (1970, J.N. Monroe) [30] describes how to detect submarines by detecting changes in surface water fluorescence caused by a submarine moving under its surface (Fig.1).

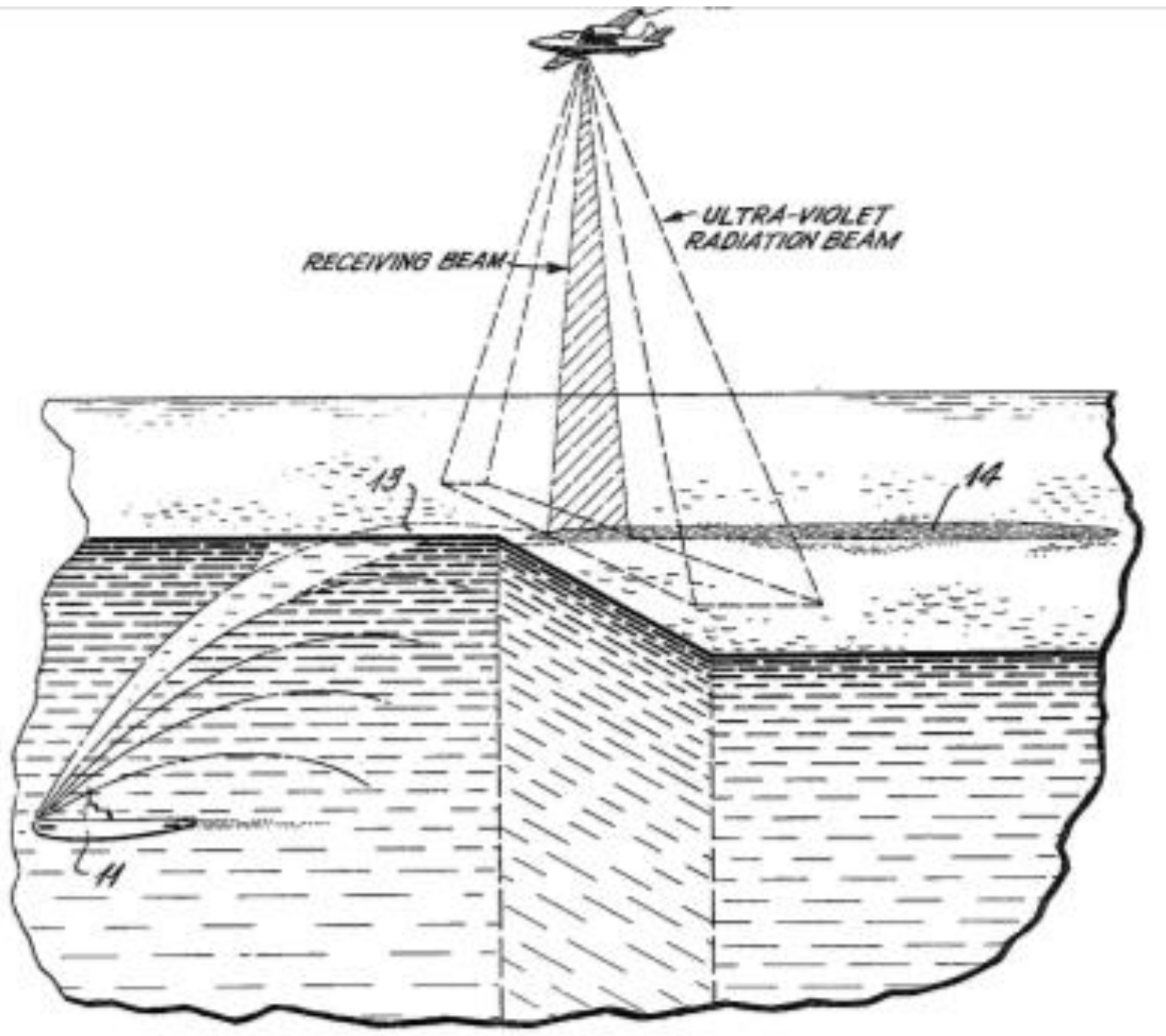

Figure. 5. The foundation of submarine sensing technique based on the optical response of the sea surface [30] 


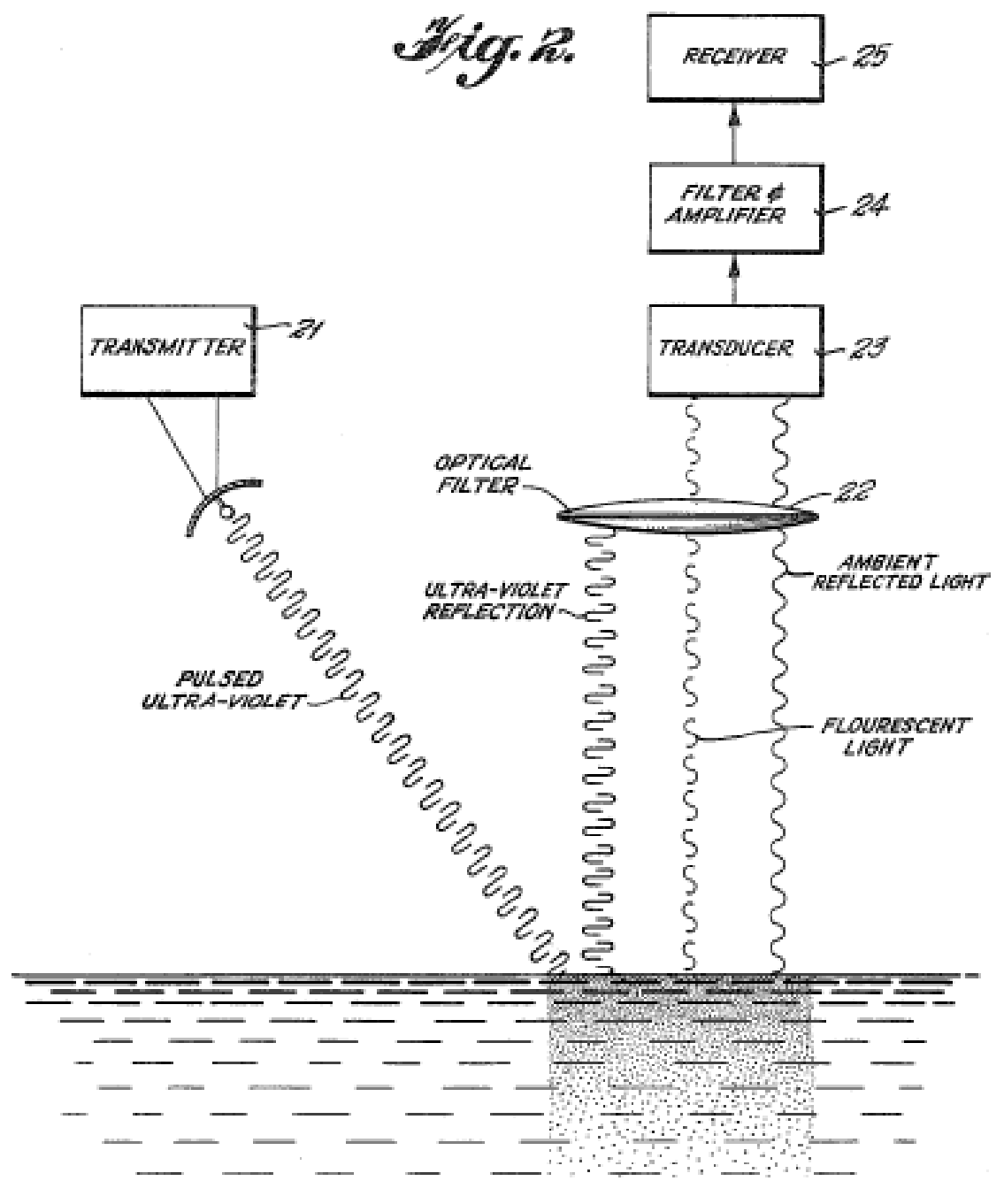

Figure. 6. The block diagram of a submarine detection device according to the method described in the invention.[30]

The foundation of this sensing technique is laid on the examination of the optical response of the surface of sea water irradiated by an ultraviolet laser beam, emitted from the board of an aircraft flying over a monitored body of water. Ultraviolet radiation irradiating the water surface energizes the fluorescent part, which will then emit visible light. The main source of this light is the fluorescence of plankton, which is particularly concentrated near the surface of water. If a submarine travels under the surface of such water, the surface concentration of the plankton changes locally and the water footprint on the water surface caused e.g. by turbulence generated by the submarine moving under the water surface can be detected from the changes in the contrast of these waters with the surrounding surface water. The fluorescent light emitted by the particles energized by ultraviolet radiation determines this contrast.

The aforementioned technique of underwater object remote-sensing is relatively effective only for objects actively moving under the surface of clean waters provided that their submersion is not very deep. It is not suitable for objects floating freely in the water (drifting). These circumstances limit the usability of the above technique to gentle weather conditions and narrow search areas which does not suffice in terms of defence against submarines.

There are some windows of opportunity in terms of underwater object remote-sensing in the optical (blue-green) range of the electromagnetic spectrum. This range is typically used for communication with submerged submarines. The usability of this optical range results from the minimum sea water absorption coefficient called Jerlov's window. 
One of the latest examples of methods of detecting submarines by optical manner is the Chinese system called "Project Guanlan" [31-33]. According to available information, the system has been officially designed to "observe great ocean waves." One of the official goals of this project was to transfer lasers capable to operate at various lengths of the optical spectrum and capable of emitting powerful laser pulses, as well as remote sensing sensors to the satellite deck, from which thermocline observations could be carried out. The thermocline is a relatively thin layer of water with the properties of reflecting sonar impulses and other acoustic signals. The acoustic properties of the thermocline create the potential for submarines to hide under it, but not before the laser beam. It is speculated that this satellite system would be able to detect submarines submerged up to about 150 meters below the water surface, but there is a view that this is rather impossible because laser radiation is generally unable to penetrate so deeply, without saying anything about water transparency. The principle of the "Project Guanlan" system is shown in Fig. 5

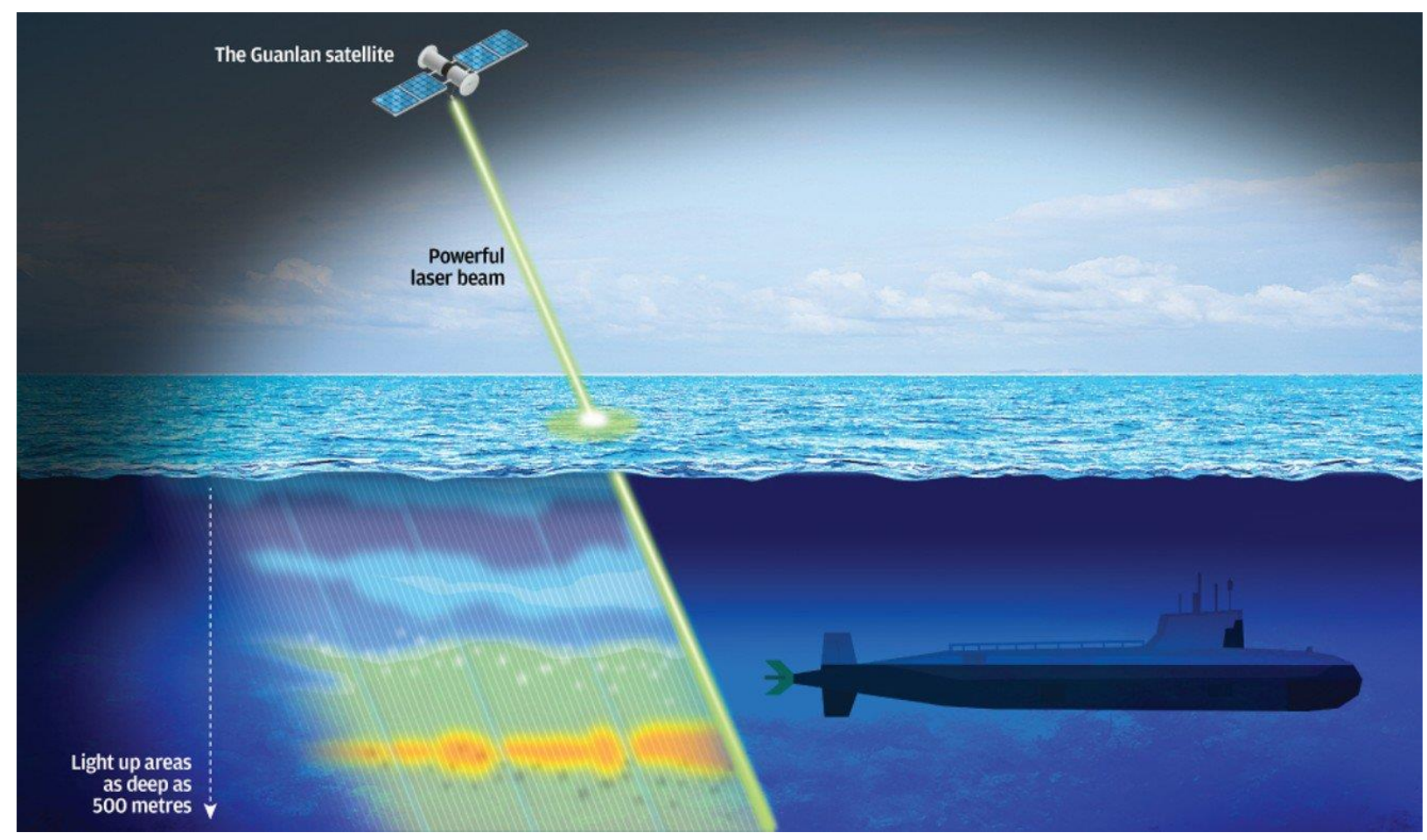

Figure. 7. Visualization of how the "Project Guanlan" system works [33]

To sum up, the possibilities of optical methods are limited by the purity of water and the condition of the sea. Contaminated, opaque, cloudy, agitated or dead waters (i.e. without plankton) do not demonstrate clear fluorescence or the presence of Jerlov's window. It may be impossible or uncertain whether optical remote sensing methods will be effective for such waters.

Patent No. CN205787179U " A kind of undersea detection system" [34] describes the underwater system of the submarine detection system. The remote sensing system includes devices that emit a laser beam, an ultrasonic sensor and a measuring apparatus. The described device emits a laser beam with a wavelength of $430-570 \mathrm{~nm}$, which is guided by in the region to be tested for submarine detection. The ultrasonic sensor is used to receive the ultrasonic wave produced after the described object absorbs the laser beam directed in its direction. The ultrasonic sensor used in the described device converts the acoustic signals generated by the laser beam into signals electrical, which are then processed and analyzed for the detection of said target. 
Thus, in the light of the problems mentioned above, the issue of effective submarine detection is still of great interest. Attempts to integrate photoacoustics into the remote sensing of underwater objects are the consequence of the aforementioned circumstances.

\section{REMOTE SENSING OF UNDERWATER OBJECTS}

One example of the use of photoacoustics in underwater remote-sensing is the OAURS (Opto Acoustic Underwater Remote Sensing) bathymetric technique [35]. The method permits measuring the depth of water bodies or the depth of immersion of objects, e.g. submarines, regardless of the state of transparency (turbidity) of the water. OASUR's principle of operation is based on optical registration of patterns that can form on the water surface due to interference of acoustic waves coming from waves reflected from elements of the surface of an underwater object. The history of research into the photoacoustic effect in liquids and its applications in developing "virtual" sources of ultrashorts underwater acoustic signals was demonstrated in the works of Egerev [36].

The source of acoustic waves probing water column are strong photoacoustic impulses generated by microsecond laser pulses fired at the water surface.

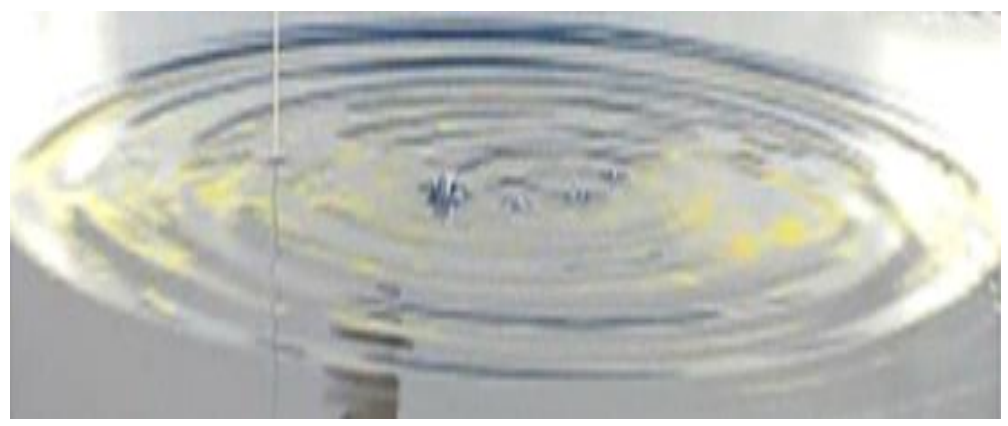

Figure. 8. An example view of the surface of the water in which the microsecond laser pulse hits. [35].

The view of water surface in which the powerful microsecond laser pulse hits water surface resembles ripples in the water surface caused, for example, by a small body falling into water (Fig. 3 ).

An example of using the underwater sound source generated by the Nd: YAG laser for air-submarine communication is shown in [37].

So, it proves that an laser pulse on the water surface (similar to a photoacoustic tomography) can generate acoustic waves propagating in a water column that can deflect as soon as they encounter a discontinuity of the medium, e.g. bottom, submerged rock or submarine. As a result of the impact of deflected acoustic waves from submerged objects on the water surface, interference patterns and microwave patterns (wrinkles) can be formed, on the basis of which it is possible to remotely detect an underwater object, regardless of the transparency of the water and the state of motion of the object.

Acoustic waves propagating in the water column deform as soon as they encounter the discontinuity of the medium, e.g. the bottom, submerged rock or submarine.

The interference patterns generated on the water surface can be optically detected from a long distance, e.g. from an aircraft or satellite (Figure 2). OAURS is a combination of LIDAR and underwater sonar - but it is based on the optical principle. 


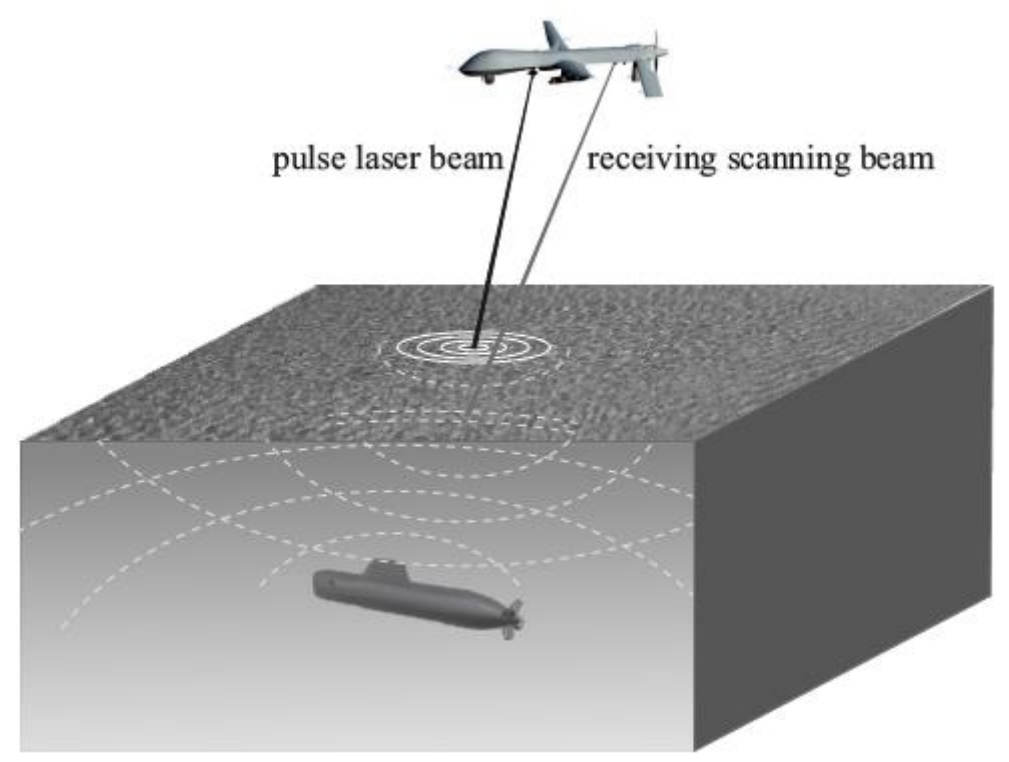

Figure 9. The principle of the OAURS device remote sensing

Interacting with the underwater object, these impulses create characteristic patterns on the water surface, based on which it is possible to detect the underwater object remotely, regardless of the transparency of the water.

An example of the configuration of the optical system of photoacoustic remote sensing is shown in Fig. 7.

The principle of optical registration of patterns that can form on the water surface due to interference of acoustic waves coming from waves deflected from elements of the surface of an underwater object.

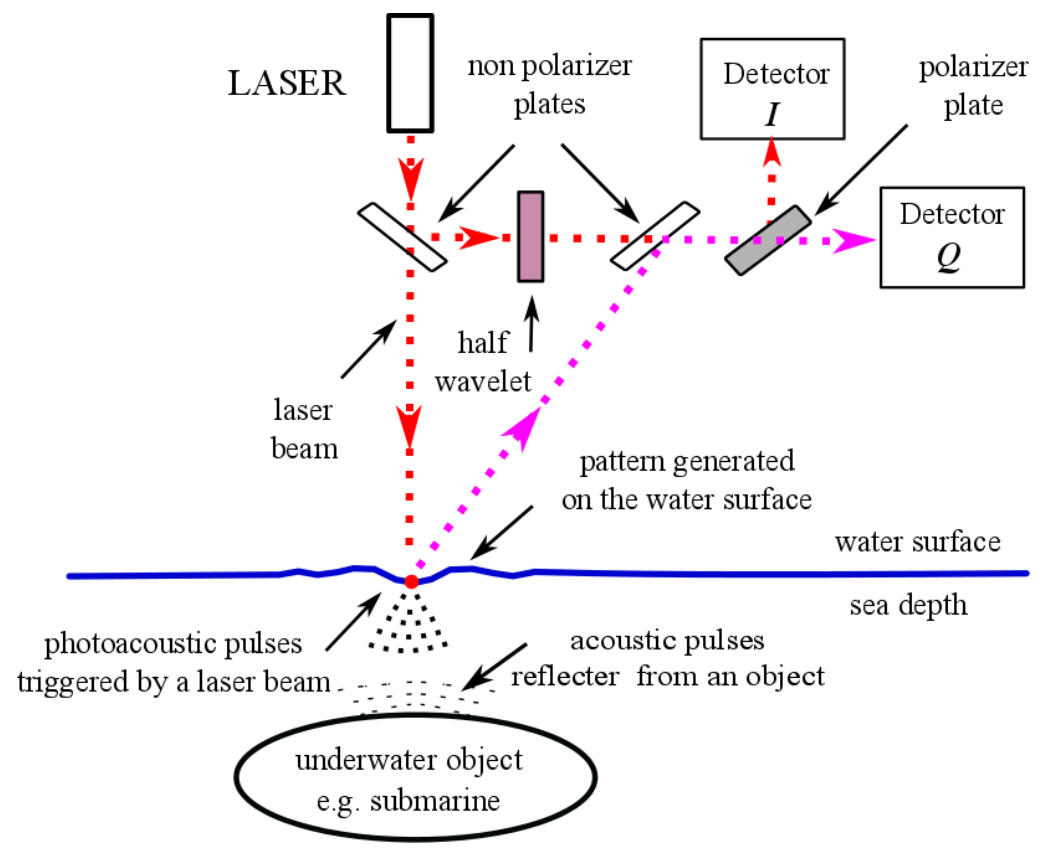

Figure. 10. Configuration of the photoacoustic underwater object remote sensing optical system 
The laser beam modulated with the echo signal of the photoacoustic impulse deflected from the object is submitted to demodulation and vector analysis. The system uses a propagator of polarized optical beams and the "I/Q" system sensing. This configuration similar to the one known in SDR receivers [39]. This structure enables the use of digital signal processing (DSP), filtering and demodulation algorithms, as well as spectral imaging of several signals at once.

\section{Conclusions}

The photoacoustic effect, which is the outcome of complex processes resulting in the production of sound waves in resilient media impacted by a modulated light stream, may be applied in photoacoustic remote sensing of materials in any state of aggregation. The materials may be transparent, dispersive, opalescent as well as light-impermeable. Photoacoustic remote sensing makes it possible to perform highly sensitive, selective and contactless measurements, e.g. to detect hazardous substances. Photoacoustic techniques open a window of opportunity for quick and unambiguous determination of the composition, concentration, surface or spatial distribution of various substances, e.g. explosives, toxic compounds, traces of biological weapons (e.g. anthrax spores), drugs, contaminants, isotopic composition, etc. They may be used for selective detection of trace concentrations - below a single ppb on solid and liquid surfaces and deep into their volume. Great flexibility, non-invasiveness and universality make photoacoustic techniques appropriate for use in practically in most tasks connected with remote sensing of biological, chemical, explosive hazard, etc., but also related to data transmission as well as ultrasonic, selective imaging of material, biological and even underwater structures [24-29].

The operating principle of the OAURS device causes that acoustic impulses generated by the laser beam, interacting with the underwater object, create characteristic patterns on the water surface, on the basis of which it is possible to remotely detect the underwater object, regardless of the transparency of the water and the state of motion of this object.

The photoacoustic imaging is frequently called photoacoustic "sonar" or "radar". This terms are convenient only that because they are related to similar result of remote sensing process, however which based not on ultrasound or radar waves emission and echo receiving but on remote sensing based on acoustic waves generated by photoacoustic effect. Basic operation principles of photoacoustic sonar /radar are similar to those used in usual sonars and radars.

\section{REFERENCES}

[1] Bell A.G., “Apparatus For Signaling And Communicating, Called „Photophone” US Patent No. 235,199 (1880).

[2] Bell A.G.," On the Production and Reproduction of Sound by Light” Am. J. of Sci. 20, 305-324(1880).

[3] Bell A.G.,"The Photophone", Science 1, 130-134 (1880).

[4] Bell A. G. i Tainter S., "Photophonic Receiver", US Patent No. 241,909 (1881).

[5] Bell A. G. i Tainter S. (1880), ,Photophone Transmitter”, US Patent No. 235,496 (1880).

[6] Röntgen W. C., "On tones produced by the intermittent irradiation of a gas", Phil. Mag. 11, 308-311 (1881).

[7] Tyndall J., "Action of an intermittent beam of radiant light upon gaseous matter" Proc. Royal Society 31, 307-317 (1881).

[8] Viegerov M.L. "New method of gas analysis based on Tyndall-Roentgen optoacoustic effect", Dokl. Akad. Nauk SSSR, 19, 687-690 (1938).

[9] Luft K. F. "Un eine Neue Methode der Registrieren den Gasanalyse mit Hilfe der Absorption Ultraroter Strahlen ohne Spektrale Zerlegung", Z. Tech. Phys., 5, 97-104 (1943),.

[10] Kosterev A. A., Bakhirkin Y.A., Curl R.F., Tittel F.K., „Quartz Tuning fork”, Opt. Lett. 27, 1902 (2002).

[11] Kosterev A. A., „Quartz -Enhanced Photoacoustic Spectroscopy”, Patent US 2005/0117155 A1.(2005)

[12] Kosterev A. A., Dong L., Thomazy D., Tittel F.K., Overby S., „QEPAS for chemical analysis of multicomponent gas mixtures". Appl Phys B Lasers and Optics, online 15 Sept. 2010.

[13] Munir, Q., Weber H. P., „Fiberoptic sensor in a resonant optoacoustic cell”, Opt. Commun. 52, 269 ().

[14] Yönak S. H. i Dowling D. R., „Parametric dependencies for photoacoustic leak localization”, J. Acoust. Soc. Am. 112, 145-155 (2002).

[15] Brassington D. J., " hoto-acoustic detection and ranging-a new technique for the remote detection of gases", J. Phys. D : Appl. Phys. 15, 219-228. (1982). 
[16] Yönak S. H. i Dowling D. R., „, Non-line-of-sight sound source localization using matched-field processing”, J. Acoust. Soc. Am. 112, 145-155 (2002).

[17] Huang E., Dowling D. R.,'High-sensitivity photoacoustic leak testing”, J. Acoust. Soc. Am. 114, 1926-1933, (2003).

[18]Ng J., Kung A. H., Miklós A, Hess P."Sensitive wavelength-modulated photoacoustic spectroscopy with a pulsed optical parametric oscillator", Opt. Lett. 29,1206-1208 (2004).

[19] Chen Z., Yang S., Xing D.,'In vivo detection of hemoglobin oxygen saturation and carboxyhemoglobin saturation with multiwavelength photoacoustic microscopy, Opt. Lett. 2012. 37 (16), 3414-3416 (2012).

[20]Lilienfeld-Toal H., Weidenmüller M., Xhelaj A., Mäntele W., „A novel approach to non-invasive glucose measurement by mid-infrared spectroscopy: The combination of quantum cascade lasers (QCL) and photoacoustic detection”, Vibrational Spectroscopy, 38, 209-215 (2005).

[21] Willer, U. \& Schade,, W. Anal Bioanal Chem (2009) 395: 275. "Photonic sensor devices for explosive detection" $<$ https://doi.org/10.1007/s00216-009-2934-2>

[22] Valentine N., Johnson T., Yin-Fong Su,.Forrester J., <https://spie.org/news/0559-rapidbacterial-identification-with-medical-and-security-applications?SSO=1> (2007).

[23] Xing Chen $\mathrm{i}$ in. „Standoff photoacoustic detection of explosives using quantum cascade laser and an ultrasensitive microphone", Applied Optics, 52, 12, (2626-2632), (2013

[24] Wang L. V, Hu S., Science. 2012 Mar 23; 335(6075): 1458-1462., "Photoacoustic Tomography: In Vivo Imaging from Organelles to Organs", $<$ https://www.nsf.gov/discoveries/disc_summ.jsp?cntn_id=135473>

[25] Xia J., Yao J.,Wang L. V. "Photoacoustic tomography: principles and advances", Electromagn Waves (Camb). 2014; 147: 1-22, < https://www.ncbi.nlm.nih.gov/pmc/ articles/PMC4311576/> ,

[26] Guedim Z., "Earthquake Detection: Undersea Fiber-Optic Cables as Acoustic Sensors", $\langle$ https://edgy.app/earthquake-detection-undersea-fiber-optic-cables-acoustic-sensors?pfrom=home\&fp=a5> c network

[27] "Undersea Fiber-Optic Cables Make Superb Seismic Network" < https://scitechdaily.com/undersea-fiber-opticcables-make-superb-seismic-network/ > (2019)

[28] Arshad, Mohd Rizal "Recent advancement in sensor technology for underwater applications" < http://nopr.niscair.res.in/handle/123456789/6203> (2009).

[29] "Large-Scale Remotely Interrogated Arrays of Fiber-Optic Interferometric Sensors for Underwater Acoustic Applications", IEEE Sensors Journal, vol. 3, no. 1, february (2003).

[30] J.N. Monroe, "Submarine detection", Patent No. US3501639 (1970).

[31] Michael Peck, "Project Guanlan",(2018).

[32] "China Is Developing A New Laser Satellite That Will Be Able To Locate U.S. Submarines 500 Meters Below The Surface 〈https://t.co/RM6qIX6BCU〉 (2018).

[33] "Project Guanlan" <https://glblgeopolitics.wordpress.com/tag/project-guanlan/> (2018).

[34]Zhou Qian, Ni Kai, Hu Kai, Wang Li Dai, Wang Xiaohao, Li Xinghui, Dong Hao, Wang Lanlan, Patent No. CN205787179U "A kind of undersea detection system" (2016).

[35] David Farrant, Jacob J. Burke, Larry. Dickinson, Philip S. Fairman, J. C. Wendoloski, "Opto-acoustic underwater remote sensing (OAURS)-an optical sonar?" Published in OCEANS'10 IEEE SYDNEY 2010

[36] Egerev S. V., "In search of a noncontact underwater acoustic source", Acoustical Phys., 49, 51-61 (2003).

[37] Duo-Min He, „High-power Nd:YAG-generated underwater sound source for air-submarine communication” Proc. V 3613, Solid State Lasers VIII; < https://doi.org/10.1117/12.347685> (1999).

[38] Reiss S.,"Photoacoustic approach shows potential to expand bioimaging's scope", <https://phys.org/news/201506-photoacoustic-approach-potential-bioimaging-scope.html> (2019)

[39] Noori BniLam, Dennis Joosens, Jan Stecke, Maarten Weyn, Conference: 13th Eur. Conf. on Antennas and Propagation, EuCap2019, Krakow/ Poland, "Low Cost AoA Unit for IoT Applications", $<$ https://www.researchgate.net/publication/332141599_Low_Cost_AoA_Unit_for_IoT_Applications> (2019).

[40] <https://www.lumasenseinc.com/EN/products/technology-overview/our-technologies/pas/photoacousticspectroscopy.html>

[41] Marra G. et al SCIENCE 2018 https://www.sciencenews.org/article/underwater-fiber-optic-cables-couldmoonlight-earthquake-sensors 